\title{
Modeling and optimization of a sugar plant using hybrid Batch Model Predictive Control
}

\author{
Michiel E. van Wissen, Joost F.C. Smeets ${ }^{*}$, Ad Muller ${ }^{* *}$ and Peter J.T. Verheijen \\ Delft University of Technology, Department of Chemical Engineering \\ Julianalaan 136, 2628 BL Delft, The Netherlands. \\ *TNO-TPD, Department of Control Engineering \\ PO Box 155, 2600 AD Delft, The Netherlands \\ **COSUN Food Technology Centre \\ Oostelijke Havendijk 15, 4704 RA Roosendaal, The Netherlands
}

\begin{abstract}
This paper presents the modeling and optimization of a sugar plant regarding heat management. The optimization has been done using Batch Model Predictive Control, which takes into account the interactions between the batch and continuous units of the plant. On the basis of the results, the development of an advice system to assist operators in the plant has been started.
\end{abstract}

\section{Introduction}

There are two reasons why model-based optimization is of high importance in the sugar industry. Using model-based optimization, one is able to predict a regular sugar quality and one can handle the large amount of energy involved. Uncertainty reduction and process restrictions motivate the choice of Model Predictive Control (MPC), a control technique that calculates a sequence of control signals in such a way that it minimizes a cost function over a prediction horizon.

In this work, the interactions between both batch and continuous units play a role, hence an adapted version of MPC is proposed, the so-called hybrid Batch-MPC. This is to distinguish from Lee et al. (1999), who used the term Batch-MPC.

An existing mass-balance model of a beet sugar factory has been extended with energy balances, recycles and variable cycle times for batch units. It is a hybrid model, consisting of batch and continuous vacuum pans and intermediate storage buffers. The product flows and volumes are calculated in the mass components: water, sugar, nonsugar and crystal. Relevant pan-program stages such as filling, seeding, boiling-up and emptying have been included.

For the extended model, control variables and process restrictions have been identified. The model has also been validated with data from last year's campaign. The model was then extended with hybrid Batch-MPC in order to optimize the energy management system.

The objective was to reduce the fluctuations in the energy system which consists of the evaporators and steamsupply for the vacuum pans. 
All of the preceding is going to be used in an operator-support system, which helps the operator in making decisions, i.e. the operator-support system is able to predict the consequence of an action taken by an operator (the system can deal with a what-if scenario).

\section{Modeling}

The sugar house and evaporation section of Suiker Unie in Groningen consists of the following units (see Figure 1). In the A, B and C-pans (also called the vacuum pans) the crystallization takes place, which are being fed by the S-1, S-A, S-B and S-C pans (also called the seed-station), in which the seed crystals used in the vacuum pans are grown. The purified juice stream passes through the evaporators (in which the solids content is typically increased from $15 \%-18 \%$ (thin juice) to $68 \%-74 \%$ (thick juice)), and subsequently through the vacuum pans. The thick juice is mixed with remelted B-and C-sugar to obtain so-called Standard Liquor which is processed in the A-pans (batch process) to end up with a solids content of typically 92-96\%, which after centrifugation leads to white sugar and A-syrup. The syrup is processed in the continuous B-pan to yield B-sugar and B-syrup which in turn is processed in the continuous C-pan and separated into $\mathrm{C}$-sugar and molasses (C-syrup). Molasses, with a solids content of $80 \%$ $85 \%$, is used in cattle-feed and in the production of alcohol. The B-and C-sugar are remelted in thin juice and returned to the A-pans.

Between the different pans, there are intermediate storage buffers, in order not to overflow a level in a certain pan. The B- and C-pans are mainly used to dispose of pollution in the juice stream. Most of the crystallization is done in the A-pans, and this happens in a batchwise manner, to have a better control of the crystal size and high supersaturations. The A- and S-pans are operated in a batchwise manner, which consists of the following steps: filling, concentrating, seeding, stabilising, boiling up, emptying steam cleaning and ready for the next strike. It should be added that the processing time of the A- and S-pans is variable, which is generally the case for batch operations (Nott and Lee, 1999).

The function of the evaporation section is twofold:

-It thickens the juice stream.

-It is used as a heat-generator for the sugarhouse and other parts of the sugar factory.

The process is modeled in Matlab/Simulink on basis of mass-balances, as in Bubnik et al. (1995). The product flows and volumes are calculated in mass components: water, sugar, non-sugar and crystal. This leads to a model with approximately 25-30 variables (e.g. levels, flows, program counters and dry substance content (brix)).

\section{Heat management}

In the original model, only mass-balances were taken into account. We introduced vapour streams between the different S-pans, A-pans and B- and C-pan, and with this we modeled the heat demand. 
In: European Symposium on COMPUTER-Aided Process ENGINEERING -12, Series:

COMPUTER-AIDED CHEMICAL ENGINEERING, 10, ED. J. GRIEVINK AND J. VAN SCHIJNDEL, 595-600 (POSTER AT ESCAPE-12, ThE Hague, The NeTHERLANDS, 26-29 MAY, 2002.)

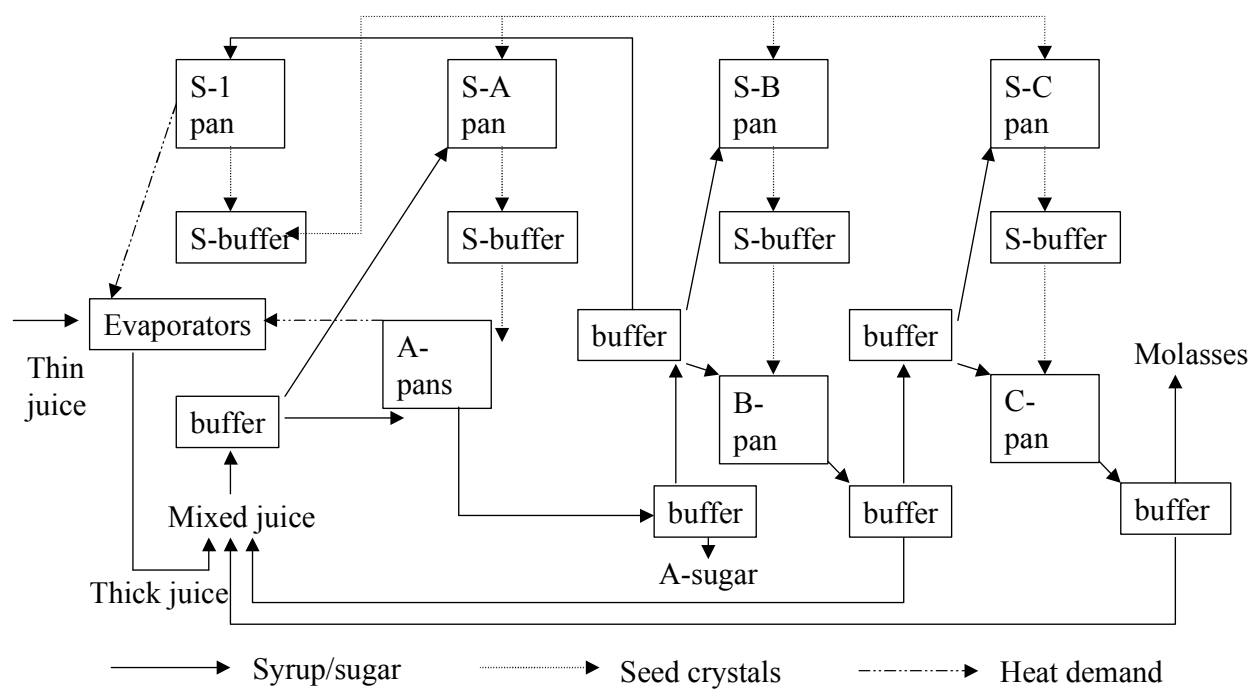

Figure 1. Units and streams of a sugarhouse and evaporation-section. Note that the heat demands of the B-, C-and S-pans are not indicated in the Figure (Bubnik et al. 1995)).

Urbaniec (1989) and Chung (2000) present a detailed description of how to model heat streams in a sugar plant.

\section{Validation}

For the model-validation we used three measurement-series (approximately with the length of one day, one week, and one month). With these data, we performed modelvalidation in two ways:

1. Model validation via mass balances.

For the modeling we used the 'one-week' measurement-data. We used checks on mass balances to verify that the 'one-day' measurement data were correct within experimental error, but not so good, when we compared it to the 'one-month' measurement-data. The main reason for this were shutdowns during that period, and it is unsure if there were losses during these shut downs

2. Model validation via measurement data.

For the three available measurements we compared the data with the model output. In order to this correctly one can compare the model results with the measurement data, just by plotting them in one plot. By doing so, one gets a fast overview if there are any discrepancies between the measurements and the model. First, we compared the datasets, and if necessary, we used a second order filter to obtain an 'average' value of a certain variable. After that, we used the obtained data set for the validation. 
A wide array of validation methods is available in literature, and most of them depend on the characteristics of the model and its intended use (Rao et al., 1998). One of the measures, that establishes that the simulation response and the data response match with a certain 'desired' tolerance (Law and Kelton, 1991) is given by

$\left[\sum_{\mathrm{i}=1}^{\mathrm{n}} \frac{\left(\mathrm{y}_{\mathrm{i}}-\mathrm{z}_{\mathrm{i}}\right)^{2}}{\sigma_{\mathrm{i}}^{2}}\right] / \mathrm{n}$

in which $y$ is the data response, $z$ is the simulation response, $n$ is the number of variables, $\sigma_{i}, i=1 \ldots n$, is the desired tolerance for the $\mathrm{i}$-th variable. The value of (1) should be close to 1 if the desired tolerance is chosen correctly. For our data we used $\sigma$ in the range of $5 \%$ to $15 \%$ depending on the estimation of the actual error.

\section{Model analysis}

After analysing the model, we came to the conclusion, that the optimization and control problem for the sugar house is not trivial because of the amount of interactions within the process:

-The interaction between the heat demand of the sugarhouse and the evaporation section is reflected in the dry substance content (brix) of the thick juice.

-Dependent on the shapes of the heat-demand-brix curves for the evaporation section and sugarhouse, the development of the thick-juice brix is intrinsically stable or unstable. An illustration of this is given in Figure 2.

Assume we have a constant available heat in the sugarhouse and evaporation-section for varying values of the brix. Suppose at a given time in the process, there is a certain heat demand to the sugar and a certain brix, represented by point A. To this point corresponds a point $\mathrm{B}$, which represents the amount of heat available to the evaporation section. Depending on the equilibrium curve of heat vs. brix in the evaporation-section, the brix of the thick juice will tend towards point $\mathrm{C}$ or point $\mathrm{D}$. This tendency repeats until the amount of heat available to the evaporation section matches the amount needed to realise the actual thick juice brix (for example point E using the heat vs. brix-curve 1, leading to a stable situation). In practice, variations in brix can be controlled by adjusting the amount of of heat to the evaporation section, the brix of thin juice to the evaporation section, or the heat demand to the sugarhouse. Hence, one of the optimization variables should be the brix of the thin juice.

- The behaviour of the process in the B- and C-pans is to a great extent dependent on the operational frequency of the A-and S-pans.

- Brix and flow of the thin juice determine the operational frequency of the A-pans and S-pans. This is important for the optimization, because the brix and flow of the thin juice are a handle to schedule the A-pans and S-pans. 
In: European SyMposium ON COMPUTER-AIDED Process ENGINEERING -12, SERIES:

COMPUTER-AIDED CHEMICAL ENGINEERING, 10, ED. J. GRIEVINK AND J. VAN

SCHIJNDEL, 595-600 (POSTER AT ESCAPE-12, ThE HAgue, THE Netherlands, 26-29

MAY, 2002.)

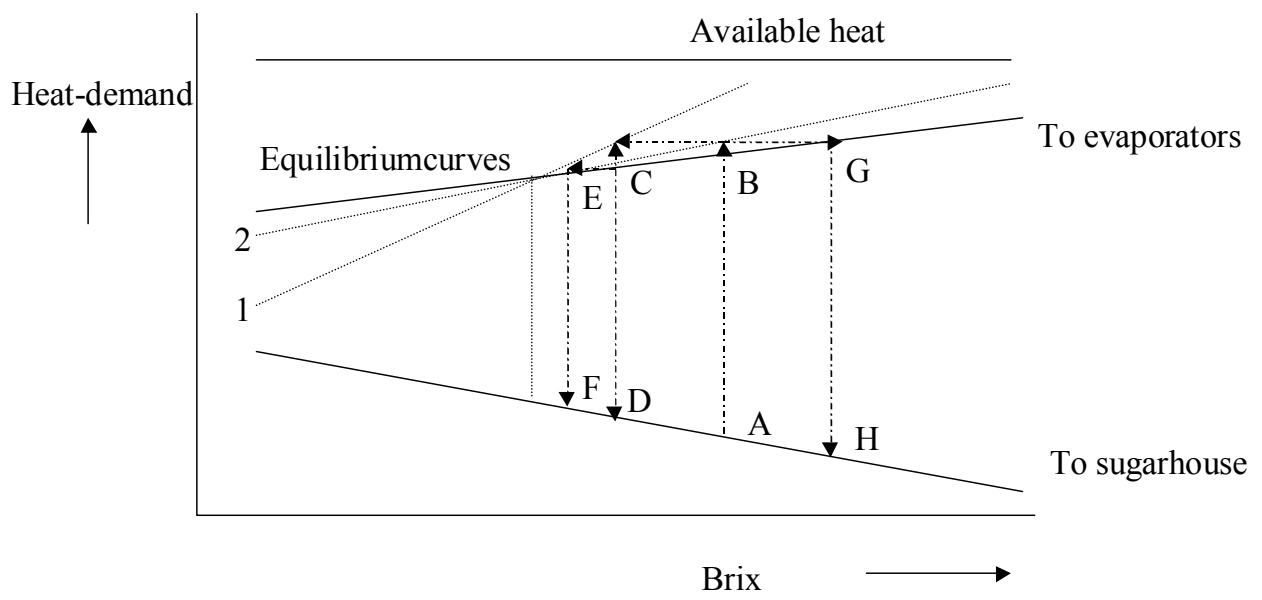

Figure 2. Brix vs. heat-demand

- A change in operation-frequencies of the A-pans leads to variations in brix of the mixed juice through. This also needs to be taken into account when developing an optimization scheme.

-The control of the flows to the B- and C-pans is not easy, because of the supply of these buffers to the S-B and S-C pans (see Figure 1). For an operator this is difficult to control manually, because every control-action taken might be good within 1-2 hours, but can lead to unforeseen actions later (say, after 8-10 hours). This is because of the long delay times in the buffers.

\section{Proposed optimization using hybrid Batch MPC}

The widespread use of Model Predictive Control (MPC) in chemical industries is because it has several nice features (see Garcia et al. (1989), Morari and Lee (1999)). MPC can be used for handling multivariable and/or constrained problems and its concepts are easy to understand for operators with only a limited knowledge of control (Bordons and Camacho, 2000).

To our knowledge no MPC-application combined with a scheduling-algorithm applied to a mixed batch/continuous system is applied. The case of scheduling a problem in a sugar factory, used to illustrate a hybrid scheduling approach, has been done by Nott and Lee (1999), but no MPC was involved here. Applications of MPC to continuous problems in a sugar plant have been reported by several authors, e.g. Bordons and Camacho (2000), Prada et al. (2000), to name but a few. In our approach, we have reduced the complex problem to a simpler problem (with less A-pans and only the relevant inputs and outputs), with all the characteristics of the more complex problem, such as its hybrid character. For this problem, the proposed objective function (OBJ) to be maximised is

$\mathrm{OBJ}=$ profit - batch costs - idle penalty - change flow penalty 
IN: EUROPEAN SyMPOSIUM ON COMPUTER-AIDED PROCESS ENGINEERING -12, SERIES:

COMPUTER-AIDED CHEMICAL ENGINEERING, 10, ED. J. GRIEVINK AND J. VAN

SCHIJNDEL, 595-600 (POSTER AT ESCAPE-12, ThE Hague, The NeTHERLANDS, 26-29

MAY, 2002.)

in which 'profit' is the turnover for the production through the system, 'batch costs' are the costs associated with beginning a batch, 'idle penalty' is the penalty for scheduling idle batch units and 'change flow penalty' is the penalty for changes in the continuous flowrate. The constraints under which the objective function OBJ (2) is maximised are

1. The model is limited to stable domains (for the thin juice brix, see Figure 2).

2. Physical constraints, such as bounds on the flows and levels.

3. Bounds on the (variable) cycle times of the A- and S-pans.

Using this objective function and constraints, the ratio between vapor input from the evaporators in the sugar mill and the steam demand of the sugarhouse is optimised.

The results of applying hybrid Batch MPC are satisfactory for the simple problem. For the more complex model we expect a significant reduction in fluctuations and heat peaks in the heat supply to the sugarhouse. Hence, we expect an increased on-spec time with tighter specs. But, more importantly, a much more stable process-operation is achieved within a time-horizon of 6-8 hours.

\section{Conclusions}

In the present paper we modeled the sugar house and evaporation-section of a sugar plant with the purpose of determing the heat streams in the process. The modeling has been done on basis of mass- and heat balances, which were checked on real plant data. After that a reduced model has been used for hybrid Batch MPC, which is a novel methodology combining scheduling and predictive control. The results are now going to be applied to the more complex model.

\section{References}

Bordons, C. and E.F. Camacho, 2000, Applications of model predictive controls in a sugar factory, Proc. ADCHEM 2000, 329.

Bubnik, Z. P. Kadlec, D. Urban and M. Bruhns, 1995, Sugar Technologists Manual.

Chung, C.C. eds., 2000, Handbook of Sugar Refining, John Wiley, New York.

Garcia, C.E., D.M. Prett and M. Morari, 1989, Model predictive control: theory and practice - a survey, Aut, 25(3), 335.

Lee, K.S., I.-S. Chin and H.J. Lee, 1999, Model predictive control technique combined with iterative learning for batch processes, AIChE J., 45(10), 2175.

Nott, P.H. and P.L.Lee, 1999, Sets formulation to schedule mixed batch/continuous process plants with variable cycle time, Comp.Chem.Eng. 23, 875.

Prada, C., C. Alonso, F. Morilla and M. Bollain, 2000, Supervision and advanced control in a beet sugar factory, Proc. ADCHEM 2000, 341.

Law, A.M., and W.D. Kelton, 1991, Simulation Modeling \& Analysis, McGraw-Hill.

Rao, L., L. Owen and D. Goldsman, 1998, Development and application of a validation framework for traffic simulation models, Proc.1998 Winter Sim.Conf.1998, 1079.

Urbaniec, K., 1989, Modern energy economy in beet sugar factories, Elsevier, Amsterdam. 